\title{
Aprendizaje-servicio, actitudes y habilidades cívicas en un grupo de estudiantes de una universidad privada de Lima, Perú
}

\section{María Méndez}

Universitat Oberta de Catalunya, España

Stefano De La Torre-Bueno

Universidad Científica del Sur, Perú

\section{Resumen}

La presente investigación explora y describe las percepciones en un grupo de estudiantes sobre la metodología y la realización de un proyecto de aprendizajeservicio llevado a cabo como trabajo final de una asignatura de la carrera de Psicología, en una universidad privada de Lima (Perú), así como los efectos en las habilidades y actitudes cívicas de los y las estudiantes. Se utilizó un diseño de métodos mixto, combinando mediciones pre y post proyecto de una versión traducida y adaptada del cuestionario de habilidades y actitudes cívicas (CASQ). Se llevaron a cabo entrevistas semi-estructuradas para recoger datos cualitativos sobre el efecto del proyecto y las percepciones de sus participantes. Los datos cuantitativos no mostraron cambios significativos en ninguna de las áreas; por el contrario, los datos cualitativos muestran una valoración positiva del proyecto de aprendizaje-servicio y de la metodología de aprendizaje, así como ciertos cambios en las actitudes cívicas de los y las estudiantes.

\section{Palabras clave}

Aprendizaje-servicio, actitudes cívicas, desarrollo de competencias, educación cívica. 


\title{
Service-learning, attitudes and civic skills in a group of students from a private university in Lima, Peru
}

\begin{abstract}
This research explores and describes the perceptions, in a group of students, about the methodology and the implementation of a Service-Learning project carried out as the final project of a subject of the Psychology degree program at a private university in Lima, Peru, as well as the effects this project had on the civic skills and attitudes of the students. A mixed methods design was used, combining pre- and post-project measurements from a translated and adapted version of the civic attitudes and skills questionnaire (CASQ). Semi-structured interviews were carried out to collect qualitative data on the effect of the project and the perceptions of its participants. The quantitative data did not show significant changes in any of the areas; on the contrary, the qualitative data show a positive assessment of the service-learning project and the learning methodology, as well as certain changes in the civic attitudes of the students.
\end{abstract}

\section{Keywords}

Service-learning, civic attitudes, skills development, civic education. 


\section{Introducción ${ }^{1}$}

La sociedad contemporánea se caracteriza por la búsqueda de soluciones a los innumerables desafíos sociales, ambientales, políticos y económicos que enfrentamos. Estos retos también se trasladan al ámbito educativo. Con los ODS (Objetivos de Desarrollo Sostenible) y la Agenda 2030 como horizonte para las sociedades, la educación, como postulan GarcíaGutiérrez et al. (2017), "es importante que no solo brinde una preparación académica de calidad, sino que también responda plenamente a las necesidades sociales, políticas y económicas de su entorno" (p.235).

Al respecto, la Agenda 2030 especifica que deben producirse cambios en nuestro estilo de vida, en el desarrollo económico y social, y en la forma en que pensamos y adquirimos conocimiento. En consecuencia, es necesario orientar la educación hacia el desarrollo de actitudes y habilidades necesarias para la formación ciudadana adaptada a las necesidades de esta nueva sociedad.

En este proceso, el papel de la Universidad es crucial, ya que es una de las instituciones encargadas de formar personas en liderazgo social. Por lo tanto, todos estos cambios sólo serán posibles con una educación que promueva la responsabilidad y la

\footnotetext{
1 Estamos particularmente agradecidas con la directora Yanira Miluska y su equipo de maestras, por su gran vocación por la educación infantil en el Perú y su colaboración en este proyecto.
}

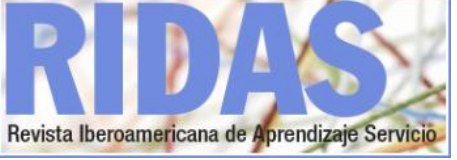

conciencia social, que prepare a los estudiantes para desenvolverse y contribuir a un mundo cada vez más complejo y cambiante (McLauchlan y Patlis, 2015), que ofrezca una preparación académica de calidad a la vez que responda a las necesidades sociales, políticas y económicas del entorno (García et al., 2017) y que esté orientada al desarrollo de actitudes y habilidades adaptadas a las necesidades de la sociedad (RuizCorbella y García-Gutiérrez, 2020).

En este contexto, surge la relevancia del uso de estrategias pedagógicas innovadoras para fomentar la adquisición de las llamadas competencias transversales. Es así, que en el último 'Informe Horizon 2019' producido por EDUCAUSE Learning Initiative (como se citó en Ruiz-Corbella y García-Gutiérrez, 2020), se hizo hincapié en la promoción del aprendizaje de forma auténtica. Es decir, favorecer metodologías educativas que sean capaces de generar experiencias prácticas, reales, donde los estudiantes aporten y construyan conocimientos, al tiempo que desarrollan las habilidades necesarias para convertirse en futuros profesionales (Ruiz-Corbella y GarcíaGutiérrez, 2020). Metodologías como el aprendizaje basado en proyectos, en competencias o el aprendizaje-servicio, capaces de crear experiencias prácticas reales donde los estudiantes aportan y construyen conocimiento, a la vez que desarrollan competencias específicas como futuros profesionales.

\footnotetext{
Méndez, M. y De La Torre-Bueno, S. (2021). Aprendizaje-servicio, actitudes y habilidades cívicas en un grupo de estudiantes de una universidad privada de Lima, Perú. RIDAS, Revista Iberoamericana de Aprendizaje Servicio, 11, 64-79. DOI10.1344/RIDAS2021.11.4
} 


\section{Marco teórico}

\subsection{Actitudes y habilidades cívicas}

La ciudadanía se puede entender como una condición o como una opción. Al hacer referencia, por un lado, a la ciudadanía como condición, se alude generalmente al lugar de nacimiento que otorga un estatus legal a los miembros de una comunidad, lo que implica el acceso a derechos y la asunción de responsabilidades. Por otro lado, al considerar la ciudadanía como una opción, es el sujeto el que se siente capaz y en libertad de decidir ser partícipe de los asuntos públicos de la comunidad a la que pertenece e intervenir en la construcción del bien común, es decir, de constituirse como ciudadano (Calmet et al., 2012). Ambas concepciones de la ciudadanía se relacionan con el ejercicio de derechos y deberes, la pertenencia a un pueblo o territorio, a un determinado nivel socioeconómico y cultural, así como la responsabilidad compartida con el entorno inmediato y con el planeta (Priegue Caamaño y Sotelio Losada, 2016).

Se entiende civismo como el área del saber, de naturaleza interdisciplinaria, que promueve valores -junto con las actitudes y comportamientos que de ellos se derivan tanto en el ámbito público como en el privado- relativos a la interacción democrática (libertad, igualdad jurídica, pluralidad y participación), al cabal cumplimiento de las obligaciones aparejadas a los distintos papeles sociales que desempeñamos (responsabilidad familiar escolar, laboral, etcétera), a la autorrealización (sujeto autónomo cognitiva y moralmente, así como felicidad), a la ayuda al más débil (solidaridad) y a la defensa de un medio ambiente saludable y sostenible (Lizcano, 2012).

En pos de desempeñar una participación efectiva en la vida cívica, se requiere el desarrollo de una variedad de habilidades y de actitudes. Por ejemplo, se espera que los jóvenes participen en procesos colectivos de toma de decisiones, resolución y negociación de conflictos, en la discusión de cuestiones políticas y sociales, o en el monitoreo de las acciones del gobierno en beneficio del interés público (Carretero et al., 2015). Las habilidades cívicas, a diferencia del conocimiento que alude a una dimensión declarativa, comprenden una dimensión procedimental que refiere a lo que las personas son capaces de hacer para una efectiva participación en la vida cívica. Estas habilidades son frecuentemente clasificadas en habilidades intelectuales, participativas y socioemocionales (Fine et al., 2007). Para los fines del presente estudio, se entienden las habilidades y actitudes cívicas como aquellas conductas $\mathrm{y} / \mathrm{o}$ capacidades cognitivas, socioemocionales y procedimentales que reflejan una vida cívica efectiva (Carretero et al., 2015).

\subsection{Aprendizaje-servicio y educación cívica}

El aprendizaje de la democracia es una parte esencial de la educación cívica. Al tratar el tema de la democracia, los formatos educativos participativos promueven el desarrollo de hábitos y actitudes democráticas por parte de los

\footnotetext{
Méndez, M. y De La Torre-Bueno, S. (2021). Aprendizaje-servicio, actitudes y habilidades cívicas en un grupo de estudiantes de una universidad privada de Lima, Perú. RIDAS, Revista Iberoamericana de Aprendizaje Servicio, 11, 64-79. DOI10.1344/RIDAS2021.11.4
} 
estudiantes, fortaleciendo su participación pública. En este contexto, se consideran los programas de aprendizaje-servicio en la escuela y a nivel universitario, instrumentos confiables para aumentar el compromiso cívico. Se considera que el aprendizaje-servicio ayuda a los estudiantes a aprender sobre instituciones políticas y democráticas. Además, genera actitudes cívicas positivas y aumenta la colaboración de los estudiantes con sus comunidades y la participación política en general (Burth, 2016).

Según expresan Puig et al. (como se citó en Puig et al., 2017) "el aprendizaje-servicio es una propuesta educativa que combina procesos de aprendizaje y de servicio a la comunidad en un solo proyecto bien articulado" (p.123), en el cual los participantes se forman al implicarse en necesidades reales del entorno con la finalidad de contribuir a su mejora. Esta práctica "incluye búsqueda de conocimientos y acciones de servicio que se enlazan según una lógica basada en la participación, la colaboración, la reflexión y la responsabilidad social" (Puig et al., 2017, 124).

En el contexto latinoamericano, según Tapia (como se citó en Tapia, 2012), el aprendizaje-servicio se suele definir como un:

"servicio solidario destinado a atender necesidades reales y sentidas de una comunidad, protagonizado activamente por los estudiantes desde el planeamiento a la evaluación, y articulado intencionadamente con los contenidos de aprendizaje (contenidos curriculares o formativos, reflexión, desarrollo de competencias para la ciudadanía y el trabajo, investigación)" (p.27).

Diversos estudios sugieren que la participación de estudiantes en programas de aprendizaje-servicio ha generado efectos positivos en diversas áreas como el involucramiento cívico, el desarrollo de habilidades sociales y el rendimiento académico (Billig, 2009; Conway et al., 2009; White, 2001). Así, por ejemplo, los estudiantes han demostrado incrementos en la internalización de estándares morales; un mayor interés, compromiso y sensibilidad hacia sus comunidades $y$ sus necesidades; y fuertes creencias en su capacidad para provocar un cambio en su entorno (Billig et al., 2005). Además, han revelado incrementos en diversas habilidades sociales relacionadas con la comunicación, liderazgo y resolución de problemas (Billig, 2009; Harwood y Radoff, 2009).

\subsection{Aprendizaje-servicio y educación cívica en el Perú}

Mientras que en Estados Unidos o Europa el aprendizaje-servicio es utilizado frecuentemente en educación superior, en muchos países de América Latina, como Perú, existen pocas experiencias que hayan evaluado los efectos del aprendizaje-servicio en el desarrollo de competencias en general, y sus efectos en las habilidades y actitudes cívicas, en particular (Díaz et al., 2016). Asimismo, existe muy poca bibliografía científica sobre ciudadanía en educación superior. Sin embargo, 
existe información local que permite justificar la importancia de ensayar nuevas metodologías para la formación de ciudadanos.

La Oficina de Medición de la Calidad de los Aprendizajes (UMC, 2004), en un estudio sobre reflexión ciudadana en estudiantes de quinto de secundaria en instituciones educativas públicas, encontró que los estudiantes tienen dificultades para asumirse como protagonistas en la toma de decisiones. Sus respuestas suelen desplazar la responsabilidad hacia los otros y revelan una poca valoración de su propio protagonismo para resolver conflictos. Por otro lado, la visión de los adultos hacia los jóvenes se relaciona con una falta de reconocimiento de ellos como ciudadanos o posibles agentes de cambio. Finalmente, los estudiantes carecen de espacios de diálogo en sus familias para hablar sobre sí mismos y sobre los asuntos públicos del país.

Más recientemente, en el estudio (Instituto Colombiano para la Evaluación de la Educación, 2016) internacional de cívica y ciudadanía (ICCS, por sus siglas en inglés: International Civic and Citizenship Education Study) realizado por la Asociación Internacional para la Evaluación del logro Académico (IEA), se buscó conocer qué tan preparados estaban los estudiantes de segundo de secundaria, de escuelas públicas y privadas del Perú, para asumir su rol como ciudadanos. El estudio manifiesta la necesidad de impulsar espacios de discusión que permitan a los estudiantes confrontar sus saberes declarativos sobre los principios democráticos a partir de prácticas cotidianas tanto en la escuela como en la comunidad. Asimismo, las instituciones educativas, como espacios de formación ciudadana, deben poder desarrollar capacidades para el análisis crítico sobre las problemáticas que afectan al sistema democrático. Por su parte, el Instituto de Estudios Peruanos (IEP, 2015) realizó un estudio sobre las percepciones de ciudadanía en distintas ciudades del Perú, en donde participaron estudiantes de sexto de primaria, de cuarto de secundaria, así como profesores y padres de familia. De manera general, se percibe al Perú como una sociedad violenta, desigual, prejuiciosa, deshonesta; en donde son los sectores más favorecidos, en términos económicos y educativos, quienes toman las decisiones; en donde se validan soluciones que ocurren por fuera de la ley, y en donde la religión ocupa espacios que deberían ser ocupados por otras organizaciones políticas.

Los resultados de estos estudios revelan no sólo la necesidad de seguir pensando el rol de las instituciones educativas formales en la formación de ciudadanos, sino de asumir esta tarea como una tarea de la sociedad en su conjunto.

Por todo lo expuesto anteriormente, la presente investigación busca explorar los efectos de un proyecto de aprendizaje-servicio en las actitudes y habilidades cívicas de un grupo de estudiantes universitarios.

De manera específica, nos preguntamos, en primer lugar, en qué medida cambian las habilidades $y$ 
actitudes cívicas de los estudiantes como resultado de la participación en un proyecto de aprendizaje - servicio. Así como, cuáles son las percepciones de las y los estudiantes sobre la experiencia de aprendizaje - servicio y los posibles efectos sobre sus actitudes y habilidades cívicas.

\section{Metodología}

\subsection{Tipo de estudio}

Para alcanzar los objetivos de la investigación, se utilizó un diseño de métodos mixtos. Creswell (2015), lo define como "un procedimiento para recolectar, analizar y mezclar métodos tanto cualitativos como cuantitativos en un solo estudio para comprender un problema de investigación" (p.537). El tipo de diseño se seleccionó en función al logro de los objetivos de la investigación, cuyo propósito es complementar los datos cualitativos y cuantitativos para obtener información más completa sobre las experiencias de participación y cambios de los estudiantes en el proyecto de aprendizaje-servicio. Como indica Walters (como se citó en Sahin y Öztürk, 2019), la investigación de métodos mixtos podría iluminar mejor los temas dentro de la investigación educativa que enfrentan controversias al investigar las complejidades de los fenómenos educativos.

\subsection{Población}

La población del estudio fueron estudiantes matriculados en dos aulas de la asignatura de psicología del desarrollo I, en una universidad privada de Lima, Perú. El número total de estudiantes matriculados en estas dos secciones fue de 63, en edades comprendidas entre los 18 y los 24 años.

A todos los estudiantes presentes se les invitó a participar en la investigación y a completar el cuestionario de habilidades y actitudes cívicas (CASQ). La totalidad de los estudiantes decidió participar en la investigación y firmaron el consentimiento informado.

Para la selección de participantes para la realización de las entrevistas, se tomaron en cuenta las calificaciones en el proyecto final. A partir de estas se elaboraron diferentes estratos ordenados de menor a mayor puntaje, y dos participantes de cada estrato fueron seleccionados de manera aleatoria. Por tanto, se utilizó un muestreo intencionado para elegir a diferentes personas que pudieran aportar sus perspectivas, para generar teoría sobre la participación en proyectos de aprendizaje-servicio, y su relación con las competencias en compromiso cívico (Creswell, 2015).

\subsection{Instrumentos}

Respecto a los instrumentos utilizados, los datos cuantitativos fueron recolectados mediante una versión traducida y adaptada al castellano y a la población del estudio, del cuestionario de habilidades y actitudes cívicas (CASQ) desarrollado por Moely et al. (2002) en Tulane University. El nivel del KMO (final) alcanzó .649 y la prueba de esfericidad de Bartlett (final) resultó significativa $p<.001$. En cuanto a la estructura de la escala adaptada alcanzó una confiabilidad de $\alpha=.836$. 
Este cuestionario está basado en medidas de aprendizaje-servicio (Astin y Sax, 1998; Eyler et al. 1997; Markus et al., 1993, como se citó en Moely et al., 2002). Se compone de seis subescalas: acción cívica, habilidades interpersonales y resolución de problemas, conciencia política, capacidad de liderazgo, justicia social y actitudes hacia la diversidad. Los autores recomiendan realizar mayor investigación utilizando entrevistas para confirmar la validez del instrumento (Moely et al., 2002).

En relación con los datos cualitativos, se utilizó una entrevista semiestructurada, con el objetivo de explorar la percepción de los estudiantes en relación con la experiencia de servicio, el proyecto de intervención, sus valoraciones del programa, la metodología utilizada y su impacto en diferentes ámbitos.

\subsection{Procedimiento}

En primer lugar, se estableció un lazo de colaboración con una institución educativa pública de Lima. Los acuerdos y responsabilidades establecidos se realizaron mediante una carta escrita firmada por la dirección de la institución y por los investigadores, creando así el "partenariado" descrito en la metodología del aprendizajeservicio (Rubio y Escofet, 2017).

Todos los estudiantes realizaron el proyecto de aprendizaje-servicio como trabajo final de la asignatura de Psicología del Desarrollo I. Se llevaron a cabo un total de seis sesiones presenciales, una vez por semana (se detalla en la tabla 1 ). Todas las actividades relacionadas con el proyecto de aprendizaje-servicio se enlazaron con los aprendizajes del sílabo de la asignatura. Se llevaron a cabo diferentes actividades, presenciales y virtuales, escritas y orales, de lectura y reflexión a lo largo de las sesiones. Los estudiantes, por grupos de 6 personas, elegían libremente qué actividades llevar a cabo en relación con los aprendizajes de la asignatura y las necesidades detectadas en una primera visita a la institución. Una vez terminadas las intervenciones se evaluó el impacto del servicio mediante una breve encuesta a la directora del centro.

Tabla 1. Sesiones del proyecto de aprendizajeservicio

\begin{tabular}{|c|c|c|}
\hline $\begin{array}{l}\text { SESIÓN DE } \\
\text { APRENDIZAJE }\end{array}$ & $\begin{array}{l}\text { OBJETIVO } \\
\text { GENERAL }\end{array}$ & ACTIVIDADES \\
\hline $\begin{array}{l}\text { SESIÓN 1: } \\
\text { SENSIBILIZACIÓN } \\
\text { Y MOTIVACIÓN }\end{array}$ & $\begin{array}{l}\text { Comprender el } \\
\text { estado de la } \\
\text { primera } \\
\text { infancia en el } \\
\text { Perú } \\
\text { identificando } \\
\text { los principales } \\
\text { problemas y } \\
\text { necesidades, } \\
\text { así como su } \\
\text { impacto en el } \\
\text { desarrollo. }\end{array}$ & $\begin{array}{l}\text { Presentar la } \\
\text { posibilidad del } \\
\text { proyecto de } \\
\text { aprendizaje- } \\
\text { servicio: la } \\
\text { idea, los } \\
\text { objetivos, cómo } \\
\text { se va a trabajar. } \\
\text { Así como } \\
\text { elaborar el } \\
\text { instrumento } \\
\text { para identificar } \\
\text { necesidades. }\end{array}$ \\
\hline $\begin{array}{l}\text { SESIÓN 2: } \\
\text { DIAGNÓSTICO DE } \\
\text { LA SITUACIÓN Y } \\
\text { DE LAS } \\
\text { NECESIDADES }\end{array}$ & $\begin{array}{l}\text { Recoger } \\
\text { información } \\
\text { tanto de la } \\
\text { institución } \\
\text { como de los } \\
\text { actores } \\
\text { involucrados, } \\
\text { sobre las } \\
\text { necesidades y } \\
\text { problemáticas, } \\
\text { a partir de los } \\
\text { instrumentos } \\
\text { elaborados. }\end{array}$ & $\begin{array}{l}\text { Identificar la } \\
\text { necesidad en la } \\
\text { que basar el } \\
\text { proyecto en } \\
\text { base a criterios } \\
\text { de viabilidad y } \\
\text { relevancia. }\end{array}$ \\
\hline
\end{tabular}




\begin{tabular}{|c|c|c|}
\hline $\begin{array}{l}\text { SESIÓN 3: } \\
\text { DELIMITACIÓN } \\
\text { DEL SERVICIO }\end{array}$ & $\begin{array}{l}\text { Diseñar una } \\
\text { propuesta de } \\
\text { proyecto en } \\
\text { base a la } \\
\text { necesidad } \\
\text { encontrada, } \\
\text { utilizando la } \\
\text { información } \\
\text { recogida en la } \\
\text { institución } \\
\text { escolar y los } \\
\text { contenidos de } \\
\text { la asignatura. }\end{array}$ & $\begin{array}{l}\text { Elaborar un plan } \\
\text { de trabajo } \\
\text { detallando las } \\
\text { actividades } \\
\text { necesarias para } \\
\text { la realización } \\
\text { del servicio. }\end{array}$ \\
\hline $\begin{array}{l}\text { SESIÓN 4: } \\
\text { EJECUCIÓN DEL } \\
\text { SERVICIO }\end{array}$ & $\begin{array}{l}\text { Realizar el } \\
\text { proyecto } \\
\text { diseñado en la } \\
\text { institución. }\end{array}$ & $\begin{array}{l}\text { Llevar a cabo } \\
\text { las actividades } \\
\text { de servicio } \\
\text { diseñadas. }\end{array}$ \\
\hline $\begin{array}{l}\text { SESIÓN 5: } \\
\text { REFLEXIÓN } \\
\text { GRUPAL }\end{array}$ & $\begin{array}{l}\text { Debatir y } \\
\text { reflexionar } \\
\text { sobre la } \\
\text { experiencia de } \\
\text { servicio, y de } \\
\text { la aplicación } \\
\text { de los } \\
\text { contenidos de } \\
\text { la asignatura. }\end{array}$ & $\begin{array}{l}\text { Reflexión en } \\
\text { torno al servicio } \\
\text { realizado, su } \\
\text { impacto y la } \\
\text { aplicación de los } \\
\text { contenidos. }\end{array}$ \\
\hline $\begin{array}{l}\text { SESIÓN 6: } \\
\text { EVALUACIÓN }\end{array}$ & $\begin{array}{l}\text { Evaluar el } \\
\text { servicio } \\
\text { realizado y la } \\
\text { experiencia } \\
\text { por medio de } \\
\text { breves } \\
\text { entrevistas a } \\
\text { los actores } \\
\text { involucrados. }\end{array}$ & $\begin{array}{l}\text { Evaluación de } \\
\text { los } \\
\text { participantes: } \\
\text { personas } \\
\text { involucradas en } \\
\text { el servicio } \\
\text { (directora, } \\
\text { maestras, etc.) } \\
\text { y estudiantes. }\end{array}$ \\
\hline
\end{tabular}

Fuente: elaboración propia

Al finalizar la última sesión del proyecto, se pidió a los estudiantes completar el cuestionario (CASQ) en una segunda oportunidad (post-test). La aplicación de las entrevistas semiestructuradas se realizó una vez finalizado el proyecto.

\subsection{Análisis de datos}

Para el análisis de datos cuantitativos, se utilizó la prueba de rangos con signo de Wilcoxon. Esta prueba permitió establecer si había diferencias o no entre las dos mediciones (pre-test y pos-test), y si estas se debían o no al azar. El análisis se realizó con la ayuda del software estadístico SPSS.

En referencia a los datos cualitativos, se realizó un análisis temático inductivo de la información recogida (Braun y Clarke, 2006). Los datos han sido codificados manualmente por los investigadores y organizados en categorías emergentes

\section{Resultados}

\subsection{Resultados cuantitativos}

El análisis de los resultados cuantitativos del cuestionario no mostró diferencia en los valores de escala del CASQ antes y después de la intervención $t=.95, p>.05$. Tampoco se evidenció diferencias cuando se comparó los valores previos y posteriores a la intervención por dimensiones: acción cívica $\mathrm{t}=-.62, \mathrm{p}>$ .05, habilidades Interpersonales y de Resolución de Problemas $t=.19, \mathrm{p}>$ .05 , conciencia Política $t=.41, p>.05$, habilidades de Liderazgo $t=-.07, p>$ .05, actitudes hacia la Diversidad $\mathrm{t}=$ $.79, p>.05$ y actitud hacia la Justicia Social disminuyó presentando un tamaño de efecto medio $\mathrm{t}=.48, \mathrm{p}<$ $.05, \mathrm{~d}$ de Cohen $=.52$.

\subsection{Resultados cualitativos}

La organización de los resultados se realizó de acuerdo con las categorías temáticas encontradas en el análisis de los datos. La información recolectada se presenta en relación con los dos ámbitos temáticos: actitudes y 
habilidades cívicas y percepciones sobre el proyecto de aprendizaje-servicio.

Dentro de estas categorías, se identificaron múltiples códigos.

Presentamos a continuación los códigos en relación con los objetivos planteados en la investigación. El primer ámbito recoge las percepciones de los y las estudiantes en torno a la participación social y la capacidad de transformación social de los y las peruanos. El segundo ámbito presenta información sobre las valoraciones de los y las estudiantes sobre la metodología utilizada y la utilidad del proyecto en la transformación de la realidad social.

Por otra parte, dentro de cada ámbito temático, los datos recogidos se agrupan en códigos de información, como sigue:

Tabla 2. Resumen del análisis cualitativo

\begin{tabular}{|c|c|}
\hline $\begin{array}{l}\text { CATEGORÍAS } \\
\text { TEMÁTICAS }\end{array}$ & Códigos \\
\hline \multirow{4}{*}{$\begin{array}{l}\text { Actitudes y } \\
\text { habilidades cívicas }\end{array}$} & $\begin{array}{l}\text { Percepción condiciones } \\
\text { sociales }\end{array}$ \\
\hline & $\begin{array}{l}\text { Percepción sobre } \\
\text { participación social }\end{array}$ \\
\hline & $\begin{array}{l}\text { Importancia de } \\
\text { involucrarse a nivel social }\end{array}$ \\
\hline & $\begin{array}{ll}\text { Capacidad } & \text { de } \\
\text { transformación social }\end{array}$ \\
\hline \multirow{5}{*}{$\begin{array}{l}\text { Percepciones del } \\
\text { proyecto de } \\
\text { aprendizaje- } \\
\text { servicio }\end{array}$} & Valoración del proyecto \\
\hline & $\begin{array}{l}\text { Relación con los y las } \\
\text { participantes }\end{array}$ \\
\hline & $\begin{array}{l}\text { Impacto positivo en el } \\
\text { entorno }\end{array}$ \\
\hline & Áreas de mejora \\
\hline & Satisfacción personal \\
\hline
\end{tabular}

Fuente: elaboración propia

\subsubsection{Actitudes y habilidades cívicas}

Su visión sobre las condiciones sociales del país muestra desigualdades en diferentes ámbitos: discriminación racial, desigualdad en el acceso a la educación, diferencias de género, etc.

- Participante 6: "de que por ejemplo todavía sigue existiendo el machismo en el Perú. Es como que 'una señorita no hace eso' $y$ bueno este, con algunas cosas de la LGTB y cosas así".

- Participante 11: "aquí hay bastante racismo, como decir a una persona cholo o negro, claro que ahora no hay mucho hacia el negro hay más hacia el cholo, porque en particular los limeños siempre se creen mucho más o tienen, o sienten que tienen algo más que las personas de provincia".

- Participante 5: "En realidad toda desigualdad es por la educación supongo yo. Entre más te educas, más surges, es más puedes conseguir mejores posiciones de trabajo".

Cuando se explora su percepción sobre la participación social en Perú, los y las participantes coinciden en que en general no hay un rol activo por parte de la población.

- Participante 2: "[...] sí, o sea no es tanta gente por lo que yo he visto pero si hay gente que si le gusta ayudar $[\ldots]^{\prime \prime}$.

- Participante 3: "No, no lo he 
visto".

- Participante 6: "[...] creo que la gente tiene que ser más sensible y ponerse más en el lugar de otras personas para entenderlos".

Aun así, coinciden en que la participación social es importante para incidir en las desigualdades del país, así como una forma de crecimiento personal.

- Participante 2: "creo que es importante porque muy aparte de que estás ayudando a otra persona creo que al ayudar a otro te hace crecer como persona".

- Participante 10: "es fundamental creo para todos es muy importante porque se ve que el Perú es un país muy bueno, hay mucha gente pobre y ayudar unos a otros sería importante".

- Participante 9: "para que de una u otra manera todos tengan una igualdad hasta en su forma de vivir por decirlo así".

Por último, al explorar sus percepciones sobre su propia capacidad para incidir en su entorno, expresan competencias o herramientas para poder realizar intervenciones.

- Participante 10: "yo pienso que tengo la capacidad de intervenir, y solucionar unos problemas que se están presentando, bueno de alguna u otra manera no sé pero si tengo la intención de intervenir".

- Participante 12: "bueno puedo juntarme con mis compañeros de la universidad, ¿no? no esperar de unirnos a un grupo sino nosotros mismos en las calles poder hacer encuestas o afiches o folletos, hacia las personas".

- Participante 5: "siento que sí, pero siento que también necesito ayuda porque uno solo no puede con todo el problema. Siento que puedo ser capaz de organizar un grupo y ver en que nos podemos centrar para solucionar los problemas".

\subsubsection{Percepciones del proyecto de aprendizaje-servicio}

Por medio de la información recolectada en las entrevistas, podemos observar en las respuestas de los y las participantes una valoración positiva sobre la metodología utilizada y el proyecto. Enfatizan en sus respuestas la utilidad de conectar los contenidos teóricos aprendidos en el aula con la práctica en un contexto real.

- Participante 5: "[...] creo que sí porque ya, o sea te dan la teoría y tú lo pones en práctica el mismo día y ves, o sea lo que te han explicado realmente lo ves en los resultados y te sientes, por lo menos en mí, me sentí: oye si esto es lo que la profesora explica".

- Participante 9: "[...] porque puedes ver lo de la clase, 
contrastarlo con la práctica y esas cosas sirven".

- Participante 11: "[...] sí, la verdad es que esto de salir a campo y hacerlo es mucho más útil creo yo que el aula".

En sus respuestas coinciden en que la relación con los y las participantes de su proyecto fue un aspecto muy satisfactorio durante la experiencia. Es la cuestión que valoran más positivamente del servicio realizado, el vínculo que establecieron con ellos y ellas.

- Participante 3: "[...]yo creo que la experiencia más valiosa fue el actuar con los niños y también hablar de ciertas problemáticas que tenían con las profesoras.

- Participante 7: "los niños cuando me vieron empezaron a cantar la canción, cómo se la supieran de memoria y de verdad me identifico mucho, sentir que realmente fue algo muy bonito porque lo que he hecho, el esfuerzo que hice, el esfuerzo que hicimos todos en grupo para que todas las dinámicas salieran bonitas valieron mucho la pena".

- Participante 12: "más que nada interactuar con los niños, eso a mí me ha cambiado un poco porque yo no tengo paciencia con los niños por así decirlo. Pero verlos a ellos, su rostro ves la necesidad de afecto o falta de atención que no les dan sus padres y me encariñe con ellos".
La metodología aprendizaje-servicio trata de poner los conocimientos adquiridos al servicio de las necesidades identificadas en un contexto, y que los contenidos curriculares del aula tengan un impacto positivo en un entorno con ciertas necesidades; en las respuestas de los y las estudiantes podemos observar que afirman haber generado un efecto positivo en el contexto del servicio. Describen que con sus conocimientos han podido hacer una diferencia.

- Participante 2: "[...] de lo que hemos hecho sí me gustó el hecho de que les dimos estrategias a la profesora para que pueda controlar los niños, y cómo ayudamos a los niños a divertirse de cierta manera $y$ olvidarse de los problemas que puedan tener en sus casas".

- Participante 8: "[...] creo que sí, sí fue útil. por ejemplo, había una dinámica mía que se llamaba el semáforo y consistía en poner un orden, cosa que la profesora nos contó que a los niños les cuesta mucho, pero en cambio con la dinámica funcionó, en cierta medida ya no era tan frecuente el desorden [...]".

- Participante 10: "yo creo que sí porque por ejemplo el tema del semáforo que aplicamos si fue útil y dio resultados porque cuando los niños estaban, o sea les explicamos en qué se basa el tema del semáforo y ellos respondieron en este ámbito, sí, me siento más motivado, por el hecho de que el trabajo nos ha 
salido bien y eso me incentiva $y$ me anima a seguir planteando más estrategias en otros ámbitos. Porque es muy importante para tratar de cambiar estas situaciones".

Cuando se indaga sobre aquellos aspectos que consideran que podrían mejorarse sobre el proyecto, los y las estudiantes coinciden en el hecho de que el proyecto debe extenderse más en el tiempo. Argumentan que, si la duración fuera mayor, podrían aumentar la calidad del servicio y por lo tanto su impacto positivo.

- Participante 5: "Le acabo de decir a la profesora de que fue muy poco tiempo el que fuimos. Con más tiempo quizás hubiera salido más bonito, más preparado, igual pienso que hemos logrado muchas cosas".

- Participante 10: "una sola vez a la semana no era suficiente, porque volver cada semana era también empezar de cero, pero solo el tiempo, que sea un poco más repetitivo, y quizás no 2 horas a la semana sino una hora pero más días".

Por último, los y las educandas expresan en sus entrevistas sentimientos de satisfacción con el servicio realizado. Destacan haber logrado el objetivo y la gratificación que les genera haber contribuido a mejorar las necesidades que presenta la institución educativa.

- Participante 5: "Sabes las condiciones de los niños quizás es un poco duro porque uno nunca sabe lo que otra familia pasa pero creo que como parte de la carrera de Psicología es muy satisfactorio saber que por lo menos has ayudado".

- Participante 6: "A favor de que lo que planeamos en grupo salió bien, que los niños aprendieron lo que tenían que aprender, y que las profesoras nos contaron $y$ confiaron en nosotros $y$ nosotros en ellas, $y$ fue todo un trabajo en grupo y entonces fue súper satisfactorio terminar esto".

- Participante 7: "[...] lo que yo he sentido con esta experiencia, de poder estar con los niños y de poder ayudar, aunque sea un poquito por la necesidad que los niños mostraban, por ejemplo de concentración, o de que a veces, se ponían un poquito inquietos $o$ no podían controlarse. Fue muy bonito, yo me sentí muy feliz y agradecida de poder ser parte de esto porque veía cómo los niños se divertían, nos hacían caso y, y al ser algo, aunque sea puedes aportar un poquito $y$ te sientes muy gratificado en hacerlo".

\section{Conclusiones}

La incorporación del aprendizajeservicio, en asignaturas de grados universitarios, fomenta vínculos de colaboración entre la universidad y la comunidad cercana, incentivando el compromiso con la sociedad, la reflexión sobre las necesidades sociales existentes y convirtiendo las prácticas 
de servicio en aprendizajes

transversales.

El aprendizaje-servicio como metodología de aprendizaje, es una forma de generar aprendizajes significativos por medio de la reflexión y dando respuesta a necesidades reales del entorno. Es por lo anterior, que impacta positivamente en la motivación académica de los y las estudiantes.

El carácter protagonista que adquieren los y las estudiantes durante el proceso de aprendizaje-servicio fomenta un sentimiento de agencia, y los empodera reconociéndose con la posibilidad de generar cambios en su entorno.

La conciencia que adquieren sobre las necesidades de la comunidad cercana por medio del diagnóstico y la reflexión que acompaña el proceso de aprendizaje-servicio, tienen un efecto en las habilidades y actitudes cívicas.

La brevedad del proyecto llevado a cabo es una de las limitaciones principales de la experiencia de aprendizaje-servicio presentada. Se recomienda realizar proyectos con una mayor duración para poder ver cambios significativos en las habilidades o actitudes cívicas.

Asimismo, con el objetivo de realizar comparaciones satisfactorias, en investigaciones con mediciones cuantitativas como en la presente, se recomienda añadir un grupo control en la comparación.

Por último, es esencial ampliar este tipo de investigaciones a una población mayor y que no se trate de experiencias aisladas. Esto permitiría extraer conclusiones mucho más veraces sobre el efecto de los proyectos de aprendizaje-servicio en los estudiantes, y en sus habilidades.

\section{Referencias bibliográficas}

Billig, S. (2009). Does quality really matter: Testing the new $\mathrm{K}-12$ servicelearning standards for quality practice. In B. E. Moely, S. H. Billig, \& B. A. Holland (Eds.), Advances in servicelearning research: Vol. 9. Creating our identities in service-learning and community engagement (pp. 131-158). Greenwich, CT: Information Age.

Billig, S., Root, S., \& Jesse, D. (2005). The relationship between quality indicators of service-learning and student outcomes: Testing the professional wisdom. In S. Root, J. Callahan, \& S. H. Billig (Eds.), Advances in service-learning research: Vol. 5. Improving service-learning practice: Research on models that enhance impacts (pp. 97-115). Greenwich, CT: Information Age.

Braun, V. y Clarke, V. (2006). Using thematic analysis in psychology. Qualitative Research in Psychology, 3(2), 77-101. doi: 10.1191/1478088706qp063oa

Burth, H. (2016). The contribution of Service-Learning programs to the promotion of civic engagement and political participation: A critical evaluation. Citizenship, Social and Economics Education, 15(1), 58-66. doi: $10.1177 / 2047173416658504$

Calmet, L., Rodríguez, M.A, y Ugarte, D. (2012). Educar para una ciudadanía 
democrática. Lima, Perú: Ministerio de Educación

Carretero, M., Haste, H., \& Bermudez, A. (2015). Civic education. En L. Corno \& E. M. Anderman (Eds.), Handbook of educational psychology (3rd ed., pp. 295-308). New York, NY: Routledge.

Conway, J., Amel, E. \& Gerwien, D. (2009). Teaching and learning in the social context: A meta-analysis of service learning's effects on academic, personal, social, and citizenship outcomes. Teaching of Psychology, 36(4), 233-245. doi:

$10.1080 / 00986280903172969$

Creswell, J. (2015). Educational research: Planning, conducting, and evaluating quantitative and qualitative research. Boston, USA : Pearson.

Díaz, K., Cárdenas, N. \& Garlock, L. (2017). Impact of Mandatory ServiceLearning Course on Civic Attitudes and Skills: Case Study in Ecuador. International Journal of Educational Excellence, (3)1, 14-38. doi: 10.18562/IJEE.021

Fine, M., Bermúdez, A., \& Barr, D. (2007). Civic learning survey.

Brookline, Massachusetts USA: FHAO.

García-Gutiérrez, J. y Ruiz-Corbella, M. (2020). Aprendizaje-servicio y tecnologías digitales: Un desafío para los espacios virtuales de aprendizaje. RIED. Revista Iberoamericana de Educación a Distancia, 23(1), 32-42. doi: $10.5944 /$ ried.23.1.25390

García-Gutiérrez, J., Ruiz-Corbella, M., \& Armentia, A. (2017). Developing Civic
Engagement in Distance Higher

Education: A Case Study of Virtual Service-Learning (vSL) Programme in Spain. Open Praxis, 9(2), 235-244. doi: 10.5944/openpraxis.9.2.578

Harwood, A. \& Radoff, S. A. (2009). Reciprocal benefits of mentoring: Results of a middle school-university collaboration. In B. E. Moely, S. H. Billig, \& B. A. Holland (Eds.), Advances in service-learning research: Vol 9. Creating our identities in servicelearning and community engagement (pp. 131-158). Greenwich, CT: Information Age.

Instituto Colombiano para la Evaluación de la Educación (2016). Estudio Internacional de Educación Cívica y Ciudadana ICCS 2016. Informe nacional para Colombia 2017. Recuperado de https://www.iea.nl/sites/default/files/2 019-

07/ICCS 2016 National Report Colom bia.pdf

Instituto de Estudios Peruanos (IEP). (2015). La ciudadanía desde la escuela: vivir en el Perú. Recuperado de https://iep.org.pe/wpcontent/uploads/2015/12/ciudadania d esde la escuela estudio encarte.pdf

Lizcano, F. (2012) Conceptos de ciudadano, ciudadanía y civismo. Polis. Revista de la Universidad Bolivariana, 11(32), Recuperado de https://www.redalyc.org/pdf/305/3052 4549014. pdf? sequence $=1$ \&isAllowed $=y$

McLauchlan, J.S. \& Patlis, L. (2015). Cultivating global citizenship in higher education: Civic engagement and service learning in joint study abroad 
courses. Revista Internacional de Educacion para la Justicia Social (RIEJS), 4(1), 111-129. doi: 10.15366/riejs2015.4.1

Moely, B., Mercer, S., Illustre, V., Miron, D. \& MacFarland, M. (2002).

Psychometric properties and correlates of the civic attitudes and skills questionnaire (CASQ): A measure of students' attitudes related to servicelearning. Michigan Journal of Community Service Learning, 8, 15-26. Recuperado de http://hdl.handle.net/2027/spo.323952 $\underline{1.0008 .202}$

Oficina de Medición de la Calidad de los Aprendizajes (UMC). (2004). Evaluación Nacional del Rendimiento Estudiantil 2004. Una primera aproximación a la evaluación de la Reflexión Ciudadana. Quinto de Secundaria. Lima: MINEDU. Recuperado de

http://umc.minedu.gob. pe/evaluacionnacional-2004/

Priegue Caamaño, D. y Sotelino Losada, A. (2016). Aprendizaje-Servicio y construcción de una ciudadanía intercultural: El proyecto PEINAS. Foro de Educación, 14(20), 361-382. doi: $10.14516 /$ fde. 2016.014 .020 .018

Puig, J., Martín, K. y Rubio, L. (2017). ¿Cómo evaluar proyectos de aprendizaje servicio? Voces de la Educación, 2(2), 122-132 Recuperado de

revista.vocesdelaeducacion.com. $\mathrm{mx} /$ ind ex.php/voces/article/view/72

Rubio, L. y Escofet, A. (Coords.) (2017). Aprendizaje-servicio (ApS): claves para su desarrollo en la
Universidad. Barcelona, España: Octaedro.

Ruiz-Corbella, M. y García-Gutiérrez, J. (2020). Aprendizaje-Servicio en escenarios digitales de aprendizaje: Propuesta innovadora en la educación superior. RIED. Revista Iberoamericana de Educación a Distancia, 23(1). doi:10.5944/ried.23.1.24391

Sahin, M. \& Öztürk, G. (2019). Mixed Method Research: Theoretical Foundations, Designs and Its Use in Educational Research. International Journal of Contemporary Educational Research, 6(2), 301-310. doi: 10.33200/ijcer.574002

Tapia, M. (2012). La solidaridad como pedagogía: la propuesta del "aprendizaje-servicio". Studium Educationis, (1), 23-36. Recuperado de:

https://ojs.pensamultimedia.it/index.ph $\mathrm{p} /$ studium/article/download/578/558/1 $\underline{784}$

White, A. (2001). Meta-analysis of service-learning research in middle and high schools (Unpublished doctoral dissertation). University of North Texas, Denton, USA.

\footnotetext{
Méndez, M. y De La Torre-Bueno, S. (2021). Aprendizaje-servicio, actitudes y habilidades cívicas en un grupo de estudiantes de una universidad privada de Lima, Perú. RIDAS, Revista Iberoamericana de Aprendizaje Servicio, 11, 64-79. DOI10.1344/RIDAS2021.11.4
} 